\title{
Sensitisation to staphylococcal enterotoxins and asthma severity: a longitudinal study in the EGEA cohort
}

\author{
Ina Sintobin ${ }^{1,10}$, Valerie Siroux ${ }^{2,10}$, Gabriële Holtappels ${ }^{1}$, Christophe Pison ${ }^{3,4}$, \\ Rachel Nadif $\mathbb{1}^{5,6}$, Jean Bousquet ${ }^{5,6,7,8}$ and Claus Bachert ${ }^{1,9}$
}

Affiliations: ${ }^{1}$ Upper Airways Research Laboratory, Ghent University, Ghent, Belgium. ${ }^{2}$ Team of Environmental Epidemiology Applied to Reproduction and Respiratory Health, Inserm, CNRS, University Grenoble Alpes, Institute for Advanced Biosciences (IAB), U1209 Joint Research Center, Grenoble, France. ${ }^{3}$ Université Grenoble Alpes, Grenoble, France. ${ }^{4}$ Clinique Universitaire de Pneumologie, Pôle Thorax et Vaisseaux, CHU de Grenoble, Inserm 1055, Grenoble, France. ${ }^{5}$ Inserm, U1168, VIMA: Aging and Chronic Diseases. Epidemiological and Public Health Approaches, Villejuif, France. ${ }^{6}$ Univ Versailles St-Quentin-en-Yvelines, UMR-S 1168, Montigny le Bretonneux, France. ${ }^{7}$ Charité - Universitätsmedizin Berlin, corporate member of Freie Universität Berlin, Humboldt-Universitätzu Berlin, and Berlin Institute of Health, Comprehensive Allergy Center, Dept of Dermatology and Allergy, Berlin, Germany. ${ }^{8}$ MACVIA-France, Fondation Partenariale FMC VIALR, Montpellier, France. ${ }^{9}$ Division of ENT Diseases, CLINTEC, Karolinska Institute, University of Stockholm, Stockholm, Sweden. ${ }^{10}$ Both authors contributed equally.

Correspondence: Claus Bachert, Upper Airways Research Laboratory, Ghent University, C Heymanslaan 10, 9000 Ghent, Belgium. E-mail: claus.bachertवugent.be

@ERSpublications

Sensitisation to Staphylococcus aureus enterotoxins (SEs) is increased in asthmatic subjects over controls; as shown for the first time, SE-IgE serum antibodies are associated with an increased subsequent risk of severe asthma and asthma exacerbations http://bit.ly/2ZjTHur

Cite this article as: Sintobin I, Siroux V, Holtappels G, et al. Sensitisation to staphylococcal enterotoxins and asthma severity: a longitudinal study in the EGEA cohort. Eur Respir J 2019; 54: 1900198 [https://doi. org/10.1183/13993003.00198-2019].

\section{ABSTRACT}

Introduction: Evidence is accumulating that Staphylococcus aureus plays an important role as disease modifier in upper and lower airway diseases. Sensitisation to S. aureus enterotoxins (SEs) was associated with an increased risk of severe asthma in previous cross-sectional studies, but evidence from longitudinal studies is lacking. We aimed to assess associations between SE-sensitisation and the subsequent risk for asthma severity and exacerbations.

Methods: This is a nested case-control study from the 20-year Epidemiological Study of the Genetics and Environment of Asthma (EGEA) cohort, including 225 adults (75 without asthma, 76 with mild asthma and 74 with severe asthma) in EGEA2 (2003-2007). For 173 of these individuals, SE-sensitisation was measured on samples collected 11 years earlier (EGEA1). Cross-sectional associations were conducted for EGEA1 and EGEA2. Longitudinal analyses estimated the association between SE-sensitisation in EGEA1 and the risk of severe asthma and asthma exacerbations assessed in the follow-up. Models were adjusted for sex, age, smoking, parental asthma/allergy and skin-prick test to house dust mite.

Results: SE-sensitisation varied between $39 \%$ in controls to $58 \%$ and $76 \%$ in mild and severe asthma, respectively, in EGEA1. An adjusted cross-sectional association showed that SE-sensitisation was associated with an increased risk of severe, but not for mild asthma. SE-sensitisation in EGEA1 was associated with severe asthma (adjusted OR 2.69, 95\% CI 1.18-6.15) and asthma exacerbations (adjusted OR 4.59, 95\% CI 1.40-15.07) assessed 10-20 years later.

Conclusion: For the first time, this study shows that being sensitised to SEs is associated with an increased subsequent risk of severe asthma and asthma exacerbations. 


\section{Introduction}

Asthma is one of the most common chronic diseases in the world; it is estimated that $\sim 300$ million people currently have asthma [1]. Trials demonstrated that guideline-defined asthma control can be attained and maintained for the majority of patients. However, asthma control is not achieved in many patients, including some patients with severe asthma despite optimal anti-inflammatory treatment [2]. Among patients with severe adult-onset asthma, comorbid conditions, including chronic rhinosinusitis (CRS) and specifically CRS with nasal polyposis, are frequent [3-5].

Staphylococcus aureus, a common human pathogen, is frequently part of the normal microflora found in the nose and on the skin [6]. S. aureus is responsible for an array of diseases and evidence is accumulating that it plays an important role as disease modifier in upper and lower airway diseases [7]. Enterotoxins from $S$. aureus (SEs) may induce polyclonal IgE formation, associated with allergic multimorbidity in adolescents [8]. The mechanisms behind the association of $S$. aureus and IgE (in tissue and in serum) have been summarised in a review [9]. Briefly, SEs can act as superantigens and are able to activate T-cells by binding to the variable $\beta$-chain of the T-cell receptors, which allows the polyclonal activation of a substantial number of T-cells. Furthermore, SEs may activate B-cells, eosinophils, epithelial cells and others, resulting in a cytokine storm locally in the tissue, and the generation of a strong inflammatory response [9].

There is an increasing body of epidemiological literature on the association between SE-sensitisation and asthma-related outcomes. Birth cohorts suggested an association between SE-sensitisation and an increased risk of persistent wheezing and asthma $[10,11]$. Moreover, S. aureus nasal colonisation was associated with increased risk of asthma prevalence, symptoms and exacerbations in children and young adults [12]. SE-sensitisation was associated with an increased risk of asthma severity, hospitalisations, oral corticosteroid use and low lung function [13]. Furthermore, specific IgE to SEs in serum (which is considered as a marker for contact with $S$. aureus and its enterotoxins) is associated with asthma Europe-wide [14], and is specifically associated with late-onset asthma in the elderly, particularly severe eosinophilic asthma with chronic rhinosinusitis comorbidity [15]. Nevertheless, the association between SE-sensitisation and an increased risk for asthma, especially severe asthma, was suggested only by cross-sectional studies [13-15] and evidence from longitudinal studies is lacking.

We aimed to analyse whether sensitisation to SEs is associated with severe asthma and asthma exacerbations later in life, using the Epidemiological Study of the Genetics and Environment of Asthma (EGEA), a cohort based on an initial group of asthma cases, first-degree relatives of cases and controls followed-up over 20 years [16-18].

\section{Materials and methods \\ Study design}

This is a nested case-control study from the EGEA cohort, a 20-year follow-up study on asthma cases, their family members and controls.

\section{Population}

The EGEA1 cohort (1991-1995) is composed of a group of 2047 participants, including 388 cases with asthma enrolled in chest clinics, 1244 first-degree relatives and 415 controls [16, 17]. Participants were recruited through a self-completed questionnaire and underwent a complete examination, including detailed questionnaires, total serum IgE, lung function tests (spirometry and methacholine challenge test), and skin-prick tests (SPT) to 11 aero-allergens (cat, Dermatophagoides pteronyssinus, Blattella germanica, olive, birch, Parieteria judaica, timothy grass, ragweed pollen, Aspergillus, Cladosporium herbarum, Alternaria tenuis). Positive SPT result was defined by a mean wheal diameter of $\geqslant 3 \mathrm{~mm}[18-20]$. $\sim 11$ years later, 1601 participants (77.1\% of the original cohort plus 58 new family members) took part in the first follow-up (EGEA2) including a detailed questionnaire and a complete examination, as performed in EGEA1 [21]. The third survey (EGEA3, 2011-2013) consisted of a self-completed questionnaire that was sent to the participants and achieved a high response rate (79.2\%) [22]. Ethical approval was obtained from the relevant institutional review board committees (Cochin Port-Royal Hospital and Necker-Enfants Malades Hospital, Paris, France). Written informed consent was signed by all participants.

For the current study, 225 participants were selected at EGEA2, including all subjects with moderate and severe asthma $(\mathrm{n}=74), 76$ subjects with mild asthma (randomly selected) and 75 control participants without asthma ever, no atopic dermatitis and no nasal polyps (defined by a negative answer to the following questions "Have you ever had atopic dermatitis?" and "Have you ever had a surgery for nasal polyps?"). Both the mild asthma group and the control group were matched on age and sex with the moderate-severe asthma group (at the group level). Specific IgE to SEs were measured in EGEA2 serum samples (see later) for these 225 selected participants; in EGEA1, serum samples for 173 of these 
participants were available for SE-sensitisation measurement. Of the 225 selected participants at EGEA2, 203 participants completed the questionnaire at EGEA3.

\section{Asthma, asthma severity and asthma exacerbation}

The definition of ever asthma was based on a positive answer to either "Have you ever had attacks of breathlessness at rest with wheezing?" or "Have you ever had asthma attacks?" or being recruited as an asthma case [17].

Asthma severity (mild versus moderate/severe) was defined at EGEA1 and EGEA2 with the available data in the study, following the principle of the Global Initiative for Asthma (GINA) 2002 guidelines, by combining the report of at least one asthma attack in the past 12 months, a clinical score and the type of asthma treatment used in the past 12 months (none, treatment not including inhaled corticosteroids (ICS), treatment including ICS). The clinical score was defined on the basis of asthma attack frequency (from 0 to 3), level of symptoms between asthma attacks (from 0 to 3), and hospitalisation for asthma over the past 12 months (yes/no); the clinical score thus ranged from 0 to 7 . Subjects were identified with "moderate-severe asthma" if they reported at least one asthma attack in the past 12 months and either 1) use of ICS treatment in the past 12 months (whatever the asthma score); 2) an asthma score $\geqslant 3$ (whatever the asthma treatment); or 3) an asthma score $=2$ and use of asthma treatment other than ICS. The other subjects were classified as "past or mild" asthma. This asthma severity classification has been used previously $[23,24]$.

The presence of at least one asthma exacerbation over the past 12 months was defined at EGEA2 and EGEA3 by the report of a hospitalisation for asthma attack or the use of oral glucocorticosteroids to help breathing any time in the past 12 months.

\section{Laboratory measurements}

Total serum IgE and SE-specific IgE (SE-mix including SE-A, SE-C and toxic shock syndrome toxin) were measured with the ImmunoCAP 100 system (Thermofisher, Uppsala, Sweden). The lower limit of SE-mix detection was set as $0.10 \mathrm{kU} \cdot \mathrm{L}^{-1}$, as in former studies $[14,25]$. The presence of SE-specific IgE in the serum is considered as a marker for exposure and an immune reaction to $S$. aureus and SEs.

\section{Statistical analysis}

Cross-sectional analyses were conducted for EGEA1 and EGEA2. To assess the risk of severe asthma associated with SE-sensitisation, multinomial logistic models were applied when considering the three-class asthma severity outcome (controls without asthma was the reference category); in analyses restricted to participants with asthma, binomial logistic models were conducted (moderate-severe asthma versus mild asthma). Models were adjusted for sex, age (years), smoking habit (never-, ex- or current smoker), parental asthma or allergy (one or more parents with asthma or allergy versus none) and SPT for house dust mite (HDM) (negative/positive).

Longitudinal analyses were conducted to assess the association between sensitisation to $S$. aureus enterotoxins in EGEA1 and the subsequent risk of 1) severe asthma at EGEA2 and 2) asthma exacerbations reported either at EGEA2 or EGEA3.

A sensitivity analysis considering the levels of SE-specific IgE was conducted $\left(<0.1 \mathrm{kU} \cdot \mathrm{L}^{-1}, 0.1-0.35 \mathrm{kU} \cdot \mathrm{L}^{-1}\right.$, $\left.>0.35 \mathrm{kU} \cdot \mathrm{L}^{-1}\right)$.

\section{Results}

Description of the study population

A description of the population in EGEA1 $(n=173)$ and EGEA2 $(n=225)$ is provided in table 1. By design, the number of participants in each group (control, past or mild asthma or moderate-severe asthma) was well balanced and there was no significant difference in mean age or sex between the three groups. No difference was observed for smoking between the three groups. In EGEA1, one-third of the moderatesevere asthma participants had a positive SPT to HDM, compared to two-thirds of the participants with mild or past asthma and $12.1 \%$ of the control participants. By definition, participants with moderatesevere asthma used significantly more inhaled corticosteroids compared to the participants with mild or past asthma and control participants $(\mathrm{p}<0.001$ at both points in time). Among the participants with asthma, $43(42.2 \%)$ and $31(20.9 \%)$ had an asthma exacerbation in the past 12 months in EGEA1 and EGEA2, respectively. Both in EGEA1 and EGEA2, the number of participants with high total IgE $\left(>100 \mathrm{kU} \cdot \mathrm{L}^{-1}\right)$ was higher in asthmatics compared to controls. In the time course between EGEA1 and EGEA2 ( $\mathrm{n}=120$ asthmatics), 47 participants had persistent mild asthma, 22 changed from mild to moderate-severe asthma, 17 changed from moderate-severe to mild asthma, and 34 had persistent moderate-severe asthma. 
TABLE 1 Population description in Epidemiological Study of the Genetics and Environment of Asthma (EGEA)1 and EGEA2

\begin{tabular}{|c|c|c|c|c|c|c|c|c|}
\hline & \multicolumn{4}{|c|}{ EGEA1 ${ }^{\#}$} & \multicolumn{4}{|c|}{ EGEA2 $?$} \\
\hline & Controls & $\begin{array}{l}\text { Past or mild } \\
\text { asthma }\end{array}$ & $\begin{array}{c}\text { Moderate-severe } \\
\text { asthma }\end{array}$ & p-value & Controls & $\begin{array}{l}\text { Past or mild } \\
\text { asthma }\end{array}$ & $\begin{array}{c}\text { Moderate-severe } \\
\text { asthma }\end{array}$ & p-value \\
\hline Subjects $\mathbf{n}$ & 69 & 59 & 45 & & 75 & 76 & 74 & \\
\hline Age years & $34.3 \pm 14.8$ & $29.6 \pm 17.3$ & $36.3 \pm 16.8$ & 0.09 & $44.8 \pm 15.3$ & $43.0 \pm 18.3$ & $42.9 \pm 16.8$ & 0.74 \\
\hline Male & 39.1 & 42.4 & 37.8 & 0.88 & 38.7 & 39.5 & 40.5 & 0.97 \\
\hline Smoking status & & & & 0.22 & & & & 0.30 \\
\hline Never-smoker & 52.5 & 56.4 & 53.9 & & 54.1 & 43.4 & 60.8 & \\
\hline Ex-smoker & 20.3 & 23.1 & 35.9 & & 25.7 & 34.2 & 21.6 & \\
\hline Current smoker & 27.1 & 20.5 & 10.3 & & 20.3 & 22.4 & 17.6 & \\
\hline$\geqslant 1$ positive $\mathrm{SPT}^{+}$ & 36.4 & 84.5 & 70.0 & $<0.001$ & 27.0 & 74.7 & 85.9 & $<0.001$ \\
\hline Positive SPT to HDM & 12.1 & 63.8 & 32.5 & $<0.001$ & 8.1 & 53.3 & 46.5 & $<0.001$ \\
\hline Total $\lg \mathrm{E}>100 \mathrm{kU} \cdot \mathrm{L}^{-1}$ & 31.9 & 76.3 & 69.8 & $<0.001$ & 22.7 & 58.7 & 70.3 & $<0.001$ \\
\hline ICS use in the past 12 months & 0 & 32.2 & 82.2 & $<0.001$ & 2.7 & 45.3 & 82.2 & $<0.001$ \\
\hline Asthma exacerbations in the past 12 months & 0 & 29.8 & 57.8 & $<0.001$ & 0 & 12.2 & 29.7 & 0.009 \\
\hline
\end{tabular}

Data are presented as mean \pm SD or $\%$, unless otherwise stated. SPT: skin-prick test; HDM: house dust mite; ICS: inhaled corticosteroid. ${ }^{\#}: \mathrm{n}=173 ;{ }^{\Uparrow}$ : $\mathrm{n}=225 ;{ }^{+}:$:11 allergens tested (cat,

Dermatophagoides pteronyssinus, Cladosporium herbarum, Alternaria tenuis, timothy grass, olive, birch, Parieteria judaica, ragweed, Aspergillus and Blattella germanical. 


\section{Cross-sectional association between SE-IgE and asthma phenotype accounting for disease severity}

In EGEA1, SE-IgE positivity varied between $39.1 \%$ in controls to $57.6 \%$ in mild asthma and $75.6 \%$ in severe asthma $(\mathrm{p}=0.006)$ (table 2$)$. SE-IgE was significantly associated with both moderate-severe asthma and mild or past asthma as compared to the control group (OR 4.81, 95\% CI 2.09-11.07 and OR 2.12, 95\% CI 1.04-4.29, respectively) (table 2). After adjustment for several confounders, SE-sensitisation was associated with a significant increased risk of moderate-severe asthma (OR 7.97, 95\% CI 2.78-22.84), but was not associated with past or mild asthma (OR 0.98, 95\% CI 0.38-2.55) (table 2). In this multiple regression model, SPT to HDM was not associated with an increased odds ratio for moderate-severe asthma (OR 2.25, 95\% CI 0.75-6.78), but increased the risk for past or mild asthma (OR 12.04, 95\% CI 4.49-32.32).

The same analysis in EGEA2 showed a similar pattern of associations, although with lower magnitudes. A statistically significant higher SE-sensitisation rate was observed in moderate-severe asthmatics (62.2\%), compared to past or mild asthmatics $(43.4 \%)$ and controls (33.3\%) (overall p=0.002) (table 2). SE-IgE positivity was significantly associated with moderate-severe asthma, but not with past or mild asthma, as compared to the control group (OR 3.29, 95\% CI 1.68-6.43 and OR 1.54, 95\% CI 0.79-2.97, respectively). The multiple logistic regression analysis showed that sensitisation to SE was associated with an increased risk of moderate-severe asthma (OR 2.09, 95\% CI 0.98-4.45), but not associated with past or mild asthma (OR 0.91, 95\% CI 0.42-1.99) (table 2). In this multiple regression model, positive SPT to HDM was positively associated in EGEA2 with both moderate-severe asthma (OR 10.53, 95\% CI 3.56-31.15) and past or mild asthma (OR 19.43, 95\% CI 6.57-57.49).

The sensitivity analysis considering the levels of SE-IgE did not show any trend for a dose-response relationship, both at EGEA1 and EGEA2 (table 3).

\section{Cross-sectional association between SE-IgE and severe asthma among subjects with asthma}

When only considering participants with asthma, and comparing participants with moderate-severe asthma to participants with past or mild asthma, SE-sensitisation was associated with a significant increased odds ratio of moderate-severe asthma in EGEA1 (OR 7.30, 95\% CI 2.22-24.01) and EGEA2 (OR 2.18, 95\% CI 1.08-4.44) (table 2). Among the cofactors included in the model, a positive SPT to HDM was associated with a decreased odds ratio for moderate-severe asthma in EGEA1 (OR 0.20, 95\% CI 0.070.59), but not in EGEA2. None of the cofactors were associated with moderate-severe asthma in EGEA2. A nonsignificant trend for a positive association between sensitisation to SEs and asthma exacerbations was observed in EGEA1 (adjusted OR 1.79, 95\% CI 0.68-4.66) and EGEA2 (adjusted OR 1.66, 95\% CI 0.69-3.96).

TABLE 2 Cross-sectional association between Staphylococcus aureus enterotoxin (SE)-IgE and asthma severity

\begin{tabular}{|c|c|c|c|c|c|c|}
\hline & \multicolumn{3}{|c|}{ EGEA1 } & \multicolumn{3}{|c|}{ EGEA2 } \\
\hline \multicolumn{7}{|l|}{ Unadjusted analysis } \\
\hline OR $(95 \% \mathrm{CI})$ & 1 & $2.12(1.04-4.29)$ & 4.81 (2.09-11.07) & 1 & $1.54(0.79-2.97)$ & $3.29(1.68-6.43)$ \\
\hline OR $(95 \% \mathrm{CI})$ & 1 & $0.98(0.38-2.55)$ & $7.97(2.78-22.84)$ & 1 & $0.91(0.42-1.99)$ & $2.09(0.98-4.45)$ \\
\hline$p$-value & & 0.97 & $<0.001$ & & 0.82 & 0.06 \\
\hline \multicolumn{7}{|l|}{ In asthmatics only } \\
\hline \multicolumn{7}{|l|}{ Unadjusted analysis } \\
\hline OR $(95 \% \mathrm{CI})$ & NA & 1 & $2.27(0.97-5.35)$ & NA & 1 & $2.14(1.11-4.11)$ \\
\hline $\mathrm{p}$-value & & & 0.06 & & & 0.02 \\
\hline
\end{tabular}

EGEA: Epidemiological Study of the Genetics and Environment of Asthma; NA: not applicable. " : models adjusted for sex, age, smoking (three classes: never-/ex-/current), parental asthma/allergy (yes/no), positive skin-prick test to house dust mite (yes/no). 
TABLE 3 Association between Staphylococcus aureus enterotoxin (SE)-IgE and asthma at Epidemiological Study of the Genetics and Environment of Asthma (EGEA)1 and EGEA2, accounting for levels of SE-specific IgE

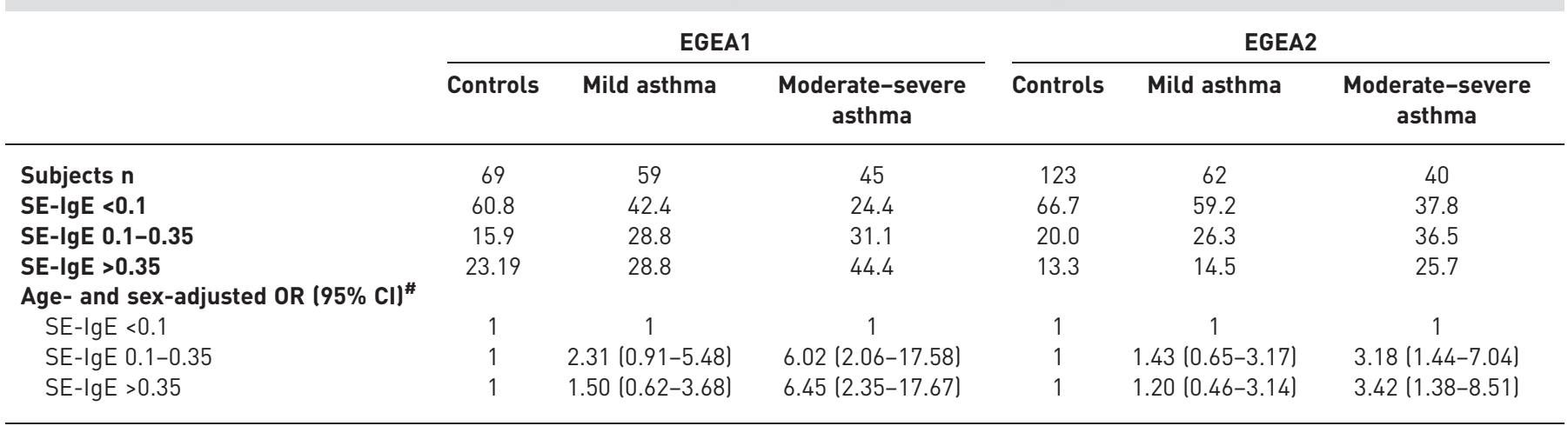

Data are presented as \%, unless otherwise stated. ${ }^{\#}$ : given that some subgroups included few subjects, cofactors in the models were restricted to age and sex.

Analysis considering change in asthma severity between EGEA1 and EGEA2 shows that the proportion of subjects with SE-sensitisation (at EGEA1 and EGEA2) was highest among those with persistent severe asthma, and persistent severe asthma was the only phenotype showing statistically significant association when compared to the persistent mild asthma group (table 4).

\section{Longitudinal association between SE-IgE at EGEA1 and severe asthma and asthma exacerbation at EGEA2 or EGEA3}

Longitudinal adjusted logistic regression analysis showed that SE-sensitisation in EGEA1 was significantly associated with moderate-severe asthma in EGEA2 (adjusted OR 2.69, 95\% CI 1.18-6.15), and a nonsignificant trend was observed for past or mild asthma (figure 1). In this multiple regression model, positive SPT to HDM in EGEA1 was significantly associated with mild or past asthma, as well as with moderate-severe asthma in EGEA2 (adjusted OR 7.80, 95\% CI 3.01-20.71 and adjusted OR 3.73, 95\% CI 1.41-9.83, respectively). Furthermore, being sensitised to SEs in EGEA1 was related to a significant increased risk of subsequent asthma exacerbations in EGEA2 or EGEA3 (adjusted- OR 4.59, 95\% CI 1.4015.07) (figure 1). Conversely, a positive SPT to HDM did not contribute to the risk of having an asthma exacerbation in the future (adjusted- OR 0.46 , 95\% CI $0.15-1.45$ ).

\section{Discussion}

This study showed that there is a significantly higher SE-sensitisation rate in severe asthmatics compared to mild asthmatics and controls. Moreover, sensitisation to SEs was associated with a strong and significantly increased risk of moderate-severe asthma, but not for past or mild asthma. When considering only participants with asthma, there was a significantly increased risk for having moderate-severe asthma when sensitised to SEs. For the first time, we were able to show longitudinal relationships between SE-sensitisation and asthma, and particularly moderate-severe asthma. The current study showed that being sensitised to SEs was a significant risk factor to have moderate-severe asthma about a decade later,

TABLE 4 Association between Staphylococcus aureus enterotoxin (SE)-IgE and change in asthma severity between Epidemiological Study of the Genetics and Environment of Asthma (EGEA)1 and EGEA2

\begin{tabular}{ccccc} 
& $\begin{array}{c}\text { Mild_EGEA1 } \\
\text { Mild_EGEA2 }\end{array}$ & $\begin{array}{c}\text { Mild_EGEA1 } \\
\text { Severe_EGEA2 }\end{array}$ & $\begin{array}{c}\text { Severe_EGEA1 } \\
\text { Mild_EGEA2 }\end{array}$ & $\begin{array}{c}\text { Severe_EGEA1 } \\
\text { Severe_EGEA2 }\end{array}$ \\
\hline SE-IgE at EGEA1 $\mathbf{n}$ & 38 & 21 & 16 & 29 \\
SE-IgE positive at EGEA1 & 57.9 & 57.1 & 68.8 & 79.3 \\
Age- and sex-adjusted OR $(95 \% \mathrm{Cl})^{\#}$ & 1 & $0.69(0.1-2.28)$ & $2.55(0.66-9.93)$ & $5.64(1.44-22.10)$ \\
SE-IgE at EGEA2 $\mathbf{n}$ & 47 & 22 & 17 & 34 \\
SE-IgE positive at EGEA2 & 46.8 & 63.6 & 47.1 & 64.7 \\
Age- and sex-adjusted OR $(95 \% \mathrm{Cl})^{\#}$ & 1 & $1.69(0.57-4.97)$ & $1.43(0.43-4.75)$ & $2.79(1.05-7.43)$ \\
\end{tabular}

Data are presented as \%, unless otherwise stated. " : given that some subgroups included few subjects, co-factors in the models were restricted to age and sex. 


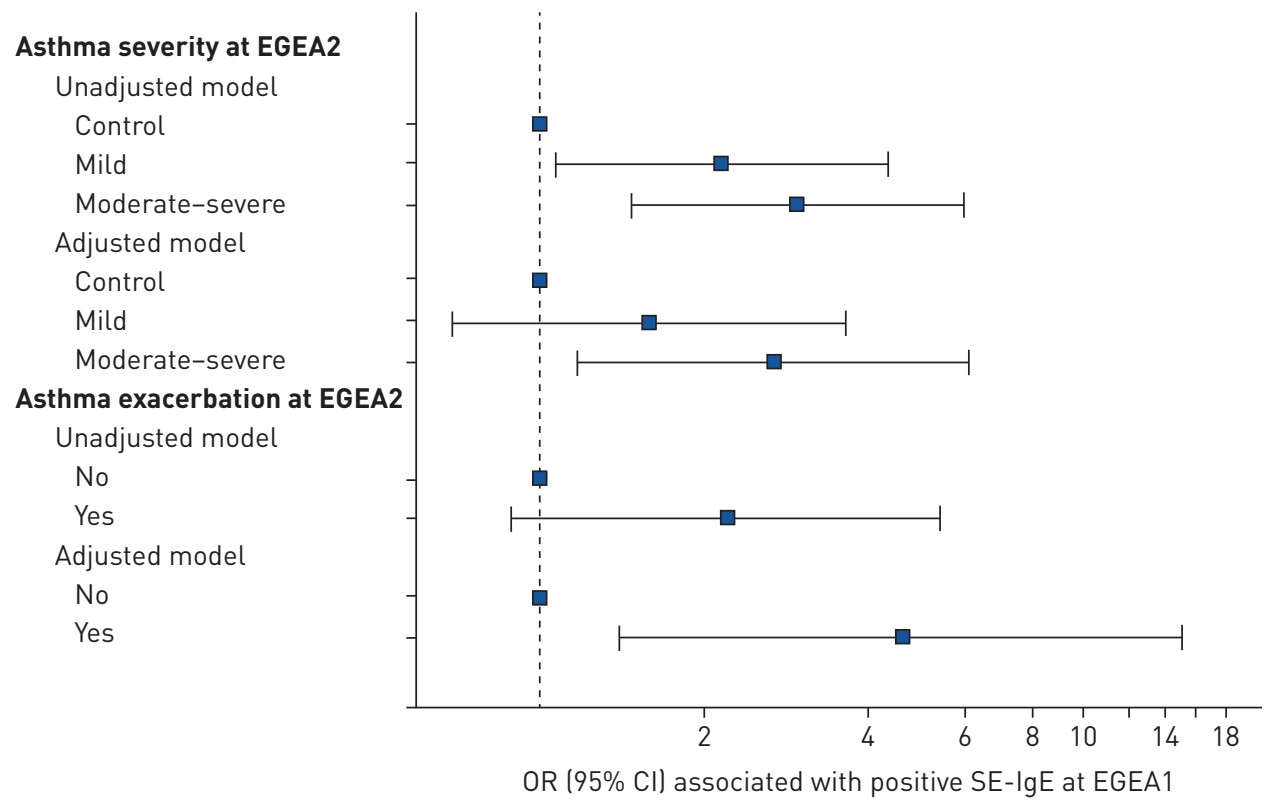

FIGURE 1 Longitudinal logistic regression analysis between Staphylococcus aureus enterotoxin (SE)-IgE in the Epidemiological Study of the Genetics and Environment of Asthma (EGEA)1 and asthma severity in EGEA2 and asthma exacerbations in EGEA2 or EGEA3 among subjects with asthma. Regression models were adjusted for sex, age, smoking (three classes: never-/ex-/current), parental asthma or allergy (yes/no), positive skin-prick test to house dust mite (yes/no).

whereas it did not contribute to the risk of having mild or past asthma. Moreover, being sensitised to SEs was a risk factor for having asthma exacerbations years later, whereas a positive SPT to HDM does not contribute to that risk.

We previously described that serum SE-IgE was associated with asthma in a large European cohort [14]. Previous reports showed a relationship between SE-sensitisation and the prevalence of asthma and/or asthma severity in different age groups. SEMic-Jusufagic et al. [10] showed an increased sensitisation rate to SEs in children with eczema and wheeze, with an increasing proportion of subjects sensitised to SE-mix with increasing symptom severity. Furthermore, we showed that asthmatics at age 20 years were more often sensitised to SEs compared to controls [26], and in an elderly Asian population, a significant association between SE-IgE and late-onset severe asthma was shown [15]. Other groups have also confirmed that increased SE-IgE levels are associated with lower respiratory function, which may be a marker for asthma severity [27]. The results of the current study are in line with these findings, but expand on these findings by showing that SE-sensitisation was specifically associated with severe asthma. This association is reinforced in the analysis considering change in asthma status over time, indicating stronger associations between SE-sensitisation and persistent severe asthma than between SE-sensitisation and severe asthma reported at a single time point, which may result from a more accurate asthma severity definition.

Although a previous epidemiological study in $>3000$ subjects showed a significant association of undefined asthma with slightly higher levels $\left(0.3 \mathrm{kU} \cdot \mathrm{L}^{-1}\right)$ of SE-IgE [14], in this study we do not show any trend for a dose-response relationship. This might be related to the fact that SE-IgE is often associated with polyclonality (induced by $S$. aureus), and thus concentrations stay on the low side.

Allergic asthma is the most common asthma phenotype in the general population of patients with asthma [28]. Nonallergic asthma is less common, typically of late onset, related to nasal polyposis and tends to be more severe [29]. BACHERT et al. [13] showed that the association between SE-IgE and severe asthma is not purely a reflection of atopy, as subjects may have negative SPT results and lack specific serum $\operatorname{IgE}$ antibodies to inhalant allergens. In this EGEA study population, 10 out of 21 (EGEA1) and 11 out of 29 patients with asthma (EGEA2) were SE monosensitised, and showed no further sensitisation. These data are in line with what was reported earlier [13] in a severe cross-sectional asthma cohort (although slightly higher), demonstrating that SE-IgE positivity (but not HDM or grass pollen IgE) was associated with asthma severity (oral steroid use, hospitalisations and lung function). We confirm these data, again separating the associations with SE-IgE from other inhalant allergen IgEs, using an independent cohort, and expand our observation with regard to prediction. Recently, proteins of S. aureus including SEs have 
been demonstrated within the sinus mucosa in nasal polyp disease [30] and have been identified as triggers for interleukin-5 release [31]. In addition, $S$. aureus proteins have been found in house dust samples [32] and may be inhaled, as $S$. aureus also belongs to the microbiome of HDM [33]. HDM may act as a carrier for antigens from bacteria and thus may cause sensitisation to both HDM and bacterial antigens [34]. Moreover, the current study also shows a high sensitisation rate to HDM in the asthmatic population; however, logistic regression models reveal a significant relationship between SE-IgE positivity and severe asthma independent from the sensitisation to HDM. Furthermore, SE-IgE positivity is associated with an increased risk of asthma exacerbations in the future, whereas a positive SPT to HDM does not contribute to that risk.

\section{Strengths and weaknesses}

The main strength of this study lies in the longitudinal nature of the data, allowing us to address first the robustness of the cross-sectional association by showing similar patterns of association in a population investigated 12 years apart, and second the role of SE-sensitisation on the subsequent risk for asthma severity and asthma exacerbations $\sim 12$ years later. Therefore, these results provide new evidence on the causality of the observed association. A further strength relates to the detailed phenotypic characterisation of the EGEA population, allowing a comprehensive asthma severity definition, adjustment for potential confounders in the association and the comparison of the magnitude of the association observed for HDM- and SE-sensitisation. Nevertheless, the association reported could be partly biased by residual for confounding, either due to missing potential confounders in the regression model (i.e. asthma comorbidities), or due to limited accuracy in the assessment of some independent variables (i.e. the smoking variable does not take into account the amount and duration of smoking).

One of the weaknesses regards the sample size of the study that lead to wide $95 \%$ confidence intervals of the estimated odds ratios and did not allow the investigation the role of SE-IgE in changes in asthma severity over time. Previous studies have shown that staphylococcal enterotoxins are strongly associated with chronic rhinosinusitis with nasal polyps, particularly in the subpopulation of patients with asthma [35]. In the current study, we only have information on "ever had surgery for nasal polyps", and only five patients with asthma at EGEA1 and 14 in EGEA2 answered yes to that question. As no nasal endoscopy was performed during clinical examination, we could not further analyse this comorbidity in our study. The analyses on asthma exacerbation rely on the report at EGEA2 or EGEA3 of oral steroids use or hospitalisation for asthma in the past 12 months. The number of asthma exacerbation from EGEA1 would have been more relevant, but this information was not available in the study. Finally, although our results rely on longitudinal data, the direction of associations, and thus causality, could be a matter of debate. ICS use is part of the definition of asthma severity and there are some concerns about long-term ICS use in terms of risk of infection, including upper and lower respiratory tract infection $[36,37]$ and mycobacterial infections [38]. While $S$. aureus is suspected to secrete enterotoxins that induce corticosteroid resistance [39], and as there is no strong support in the literature that ICS act as an immunomodulator increasing the risk of $S$. aureus, we cannot totally avoid this possible effect.

\section{Conclusion}

This study confirms previous findings of a higher SE-sensitisation rate in severe asthmatics compared to mild asthmatics or controls. Being sensitised to SEs is significantly associated with an increased risk for having moderate-severe asthma, whereas having a positive SPT to HDM was associated with a decreased risk for having severe asthma. Moreover, for the first time, we showed using longitudinal data that being sensitised to SEs is a significant risk factor for having asthma exacerbation years later, whereas being sensitised to HDM does not contribute to that risk.

Acknowledgements: The authors thank all those who participated in the setting of the EGEA study and on the examinations of the individuals. The authors are grateful to the three CIC-Inserm of Necker, Grenoble and Marseille who supported the EGEA study and in which participants were examined. They are indebted to all the individuals who participated in the EGEA study without whom the study would not have been possible. We thank the Epidemiological Study on Genetics and Environment of Asthma (EGEA) cooperative group members as follows. Coordination: V. Siroux (epidemiology, PI since 2013); F. Demenais (genetics); I. Pin (clinical aspects); R. Nadif (biology); F. Kauffmann (PI 1992-2012). Respiratory epidemiology: Inserm ex-U 700, Paris: M. Korobaeff (EGEA1), F. Neukirch (EGEA1); Inserm ex-U 707, Paris: I. Annesi-Maesano (EGEA1-2); Inserm ex-U 1018, Villejuif: F. Kauffmann, M.P. Oryszczyn (EGEA1-2); Inserm U 1168, Villejuif: N. Le Moual, R. Nadif, R. Varraso; Inserm U 1209 Grenoble: V. Siroux. Genetics: Inserm ex-U 393, Paris: J. Feingold; Inserm U 946, Paris: E. Bouzigon, F. Demenais, M.H. Dizier; CNG, Evry: I. Gut (now CNAG, Barcelona, Spain), M. Lathrop (now McGill University, Montreal, Canada). Clinical centres: Grenoble: I. Pin, C. Pison; Lyon: D. Ecochard (EGEA1), F. Gormand, Y. Pacheco; Marseille: D. Charpin (EGEA1), D. Vervloet (EGEA1-2); Montpellier: J. Bousquet; Paris Cochin: A. Lockhart (EGEA1), R. Matran (now in Lille); Paris Necker: E. Paty (EGEA1-2), P. Scheinmann (EGEA1-2); Paris-Trousseau: A. Grimfeld (EGEA1-2), J. Just. Data and quality management: Inserm ex-U155 (EGEA1): J. Hochez; Inserm U 1168, Villejuif: N. Le Moual; Inserm ex-U780: C. Ravault (EGEA1-2); Inserm ex-U794: N. Chateigner (EGEA1-2); Grenoble: J. Quentin (EGEA1-2). 
Support statement: C. Bachert is supported by the Sixth European Union Framework programme for research, contract number FOOD-CT-2004-506378, and the Seventh EU Framework programme, grant agreement number 260895 (PREDICTA); from the Flemish Scientific Research Board, FWO, projects 1841713N, G.039412N, G.067512N; BOF14/ GOA/019; BOF01J01113). EGEA data collection was funded in part by hospital programme of clinical research (PHRC)-Paris, PHRC-Grenoble, national PHRC 2012, scientific committee "AGIR pour les Maladies Chroniques", Merck Sharp and Dohme (MSD) and the GA2LEN project (Global Allergy and Asthma European Network). Funding information for this article has been deposited with the Crossref Funder Registry.

Conflict of interest: V. Siroux reports personal fees for lecturing from AstraZeneca, outside the submitted work. G. Holtappels has nothing to disclose. C. Pison reports non-financial support for meeting attendance from GSK France, AZ France, Boehringer Ingelheim and Novartis, outside the submitted work. R. Nadif has nothing to disclose. J. Bousquet reports personal fees for advisory board work, consultancy and lecturing from Chiesi, Cipla, Hikma,

Menarini, Mundipharma, Mylan, Novartis, Sanofi-Aventis, Takeda, Teva and Uriach, and owns shares in KYomed-INNOV, outside the submitted work. C. Bachert has nothing to disclose. I. Sintobin has nothing to disclose.

\section{References}

1 Masoli M, Fabian D, Holt S, et al. The global burden of asthma: executive summary of the GINA Dissemination Committee report. Allergy 2004; 59: 469-478.

2 Braido F. Failure in asthma control: reasons and consequences. Scientifica 2013; 2013: 549252.

3 Bresciani M, Paradis L, Des Roches A, et al. Rhinosinusitis in severe asthma. J Allergy Clin Immunol 2001; 107: 73-80.

4 ten Brinke A, Grootendorst DC, Schmidt JT, et al. Chronic sinusitis in severe asthma is related to sputum eosinophilia. J Allergy Clin Immunol 2002; 109: 621-626.

5 Denlinger LC, Phillips BR, Ramratnam S, et al. Inflammatory and comorbid features of patients with severe asthma and frequent exacerbations. Am J Respir Crit Care Med 2017; 195: 302-313.

6 Huvenne W, Hellings PW, Bachert C. Role of staphylococcal superantigens in airway disease. Int Arch Allergy Immunol 2013; 161: 304-314.

7 Zhang N, Holtappels G, Gevaert P, et al. Mucosal tissue polyclonal IgE is functional in response to allergen and SEB. Allergy 2011; 66: 141-148.

8 Sørensen M, Klingenberg C, Wickman M, et al. Staphylococcus aureus enterotoxin sensitization is associated with allergic poly-sensitization and allergic multimorbidity in adolescents. Allergy 2017; 72: 1548-1555

9 Bachert C, Zhang N. Chronic rhinosinusitis and asthma: novel understanding of the role of IgE 'above atopy'. J Intern Med 2012; 272: 133-143.

10 Semic-Jusufagic A, Bachert C, Gevaert P, et al. Staphylococcus aureus sensitization and allergic disease in early childhood: population-based birth cohort study. J Allergy Clin Immunol 2007; 119: 930-936.

11 Hollams EM, Hales BJ, Bachert C, et al. Th2-associated immunity to bacteria in teenagers and susceptibility to asthma. Eur Respir J 2010; 36: 509-516.

12 Davis MF, Peng RD, McCormack MC, et al. Staphylococcus aureus colonization is associated with wheeze and asthma among US children and young adults. J Allergy Clin Immunol 2015; 135: 811-813.

13 Bachert C, van Steen K, Zhang N, et al. Specific IgE against Staphylococcus aureus enterotoxins: an independent risk factor for asthma. J Allergy Clin Immunol 2012; 130: 376-381.

14 Tomassen P, Jarvis D, Newson R, et al. Staphylococcus aureus enterotoxin-specific IgE is associated with asthma in the general population: a GA ${ }^{2}$ LEN study. Allergy 2013; 68: 1289-1297.

15 Song WJ, Sintobin I, Sohn KH, et al. Staphylococcal enterotoxin IgE sensitization in late-onset severe eosinophilic asthma in the elderly. Clin Exp Allergy 2016; 46: 411-421.

16 Kauffmann F, Dizier MH. EGEA (Epidemiological study on the Genetics and Environment of Asthma, bronchial hyperresponsiveness and atopy) - design issues. EGEA Co-operative Group. Clin Exp Allergy 1995; 25: Suppl. 2, $19-22$.

17 Kauffmann F, Dizier MH, Pin I, et al. Epidemiological study of the genetics and environment of asthma, bronchial hyperresponsiveness, and atopy: phenotype issues. Am J Respir Crit Care Med 1997; 156: S123-S129.

18 Kauffmann F, Dizier MH, Annesi-Maesano I, et al. EGEA (Epidemiological study on the Genetics and Environment of Asthma, bronchial hyperresponsiveness and atopy) - descriptive characteristics. Clin Exp Allergy 1999; 29: Suppl. 4, 17-21.

19 Boudier A, Curjuric I, Basagaña X, et al. Ten-year follow-up of cluster-based asthma phenotypes in adults. A pooled analysis of three cohorts. Am J Respir Crit Care Med 2013; 188: 550-560.

20 Siroux V, Boudier A, Bousquet J, et al. Asthma control assessed in the EGEA epidemiological survey and health-related quality of life. Respir Med 2012; 106: 820-828.

21 Siroux V, Boudier A, Bousquet J, et al. Phenotypic determinants of uncontrolled asthma. J Allergy Clin Immunol 2009; 124: 681-687.

22 Epidemiological study on the Genetics and Environment of Asthma, bronchial hyperresponsiveness and atopy. EGEA: General Presentation 2013. https://egeanet.vjf.inserm.fr/index.php/en/presentation-en/design Date last updated: 26 September, 2016.

23 Le Moual N, Siroux V, Pin I, et al. Asthma severity and exposure to occupational asthmogens. Am J Respir Crit Care Med 2005; 172: 440-445.

24 Varraso R, Siroux V, Maccario J, et al. Asthma severity is associated with body mass index and early menarche in women. Am J Respir Crit Care Med 2005; 171: 334-339.

25 Bachert C, Gevaert P, Howarth P, et al. IgE to Staphylococcus aureus enterotoxins in serum is related to severity of asthma. J Allergy Clin Immunol 2003; 111: 1131-1132.

26 Sintobin I, Keil T, Lau S, et al. Is immunoglobulin E to Staphylococcus aureus enterotoxins associated with asthma at 20 years? Pediatr Allergy Immunol 2015; 26: 461-465.

27 Kowalski ML, Cieślak M, Pérez-Novo CA, et al. Clinical and immunological determinants of severe/refractory asthma (SRA): association with staphylococcal superantigen-specific IgE antibodies. Allergy 2011; 66: 32-38.

28 Schatz M, Rosenwasser L. The allergic asthma phenotype. J Allergy Clin Immunol Pract 2014; 2: 645-648. 
29 Barnes PJ. Intrinsic asthma: not so different from allergic asthma but driven by superantigens? Clin Exp Allergy 2009; 39: 1145-1151.

30 Schmidt F, Meyer T, Sundaramoorthy N, et al. Characterization of human and Staphylococcus aureus proteins in respiratory mucosa by in vivo- and immunoproteomics. J Proteomics 2017; 155: 31-39.

31 Bachert C, Holtappels G, Merabishvili M, et al. Staphylococcus aureus controls interleukin-5 release in upper airway inflammation. J Proteomics 2018; 180: 53-60.

32 Davis MF, Ludwig S, Brigham EP, et al. Effect of home exposure to Staphylococcus aureus on asthma in adolescents. J Allergy Clin Immunol 2018; 141: 402-405.

33 Chan TF, Ji KM, Yim AK, et al. The draft genome, transcriptome, and microbiome of Dermatophagoides farinae reveal a broad spectrum of dust mite allergens. J Allergy Clin Immunol 2015; 135: 539-548.

34 Dzoro S, Mittermann I, Resch-Marat Y, et al. House dust mites as potential carriers for IgE sensitization to bacterial antigens. Allergy 2018; 73: 115-124.

35 Bachert C, Zhang N, Holtappels G, et al. Presence of IL-5 protein and IgE antibodies to staphylococcal enterotoxins in nasal polyps is associated with comorbid asthma. J Allergy Clin Immunol 2010; 126: 962-968.

36 Yang $\mathrm{M}$, Zhang $\mathrm{Y}$, Chen $\mathrm{H}$, et al. Inhaled corticosteroids and risk of upper respiratory tract infection in patients with asthma: a meta-analysis. Infection 2019; 47: 377-385.

37 McKeever T, Harrison TW, Hubbard R, et al. Inhaled corticosteroids and the risk of pneumonia in people with asthma: a case-control study. Chest 2013; 144: 1788-1794.

38 Brode SK, Campitelli MA, Kwong JC, et al. The risk of mycobacterial infections associated with inhaled corticosteroid use. Eur Respir J 2017; 50: 1700037.

39 Uong P, Curran-Everett D, Leung DYM. Staphylococcus aureus colonization is associated with increased inhaled corticosteroid requirements in patients with atopic dermatitis and asthma. J Allergy Clin Immunol Pract 2017; 5: $1782-1783$. 